\title{
Correlation Between Central Corneal Thickness and Radial Peripapillary Capillary Density, in Patients With Ocular Hypertension
}

\author{
Elpida Kollia ${ }^{1}$, Eleni Patsea ${ }^{2}$, Ilias Georgalas ${ }^{1}$, Dimitrios Brouzas ${ }^{3}$, Dimitrios Papaconstantinou ${ }^{3}$ \\ 1. Ophthalmology, National and Kapodistrian University of Athens School of Medicine, Athens, GRC 2. \\ Ophthalmology/Glaucoma, Ophthalmiatreion Athinon, Athens, GRC 3. Ophthalmology, "G. Gennimatas" Hospital, \\ National and Kapodistrian University of Athens School of Medicine, Athens, GRC
}

Corresponding author: Elpida Kollia, elpidakoll@gmail.com

\section{Abstract}

\section{Purpose}

To investigate any possible relationship between the central corneal thickness and the radial peripapillary capillary density detected by optical coherence tomography (OCT) angiography in eyes with ocular hypertension.

\section{Materials and methods}

In this observational study, 135 eyes were examined. OCT angiography of the optic disc ( $4.5 \mathrm{~mm}$ ) and ultrasound corneal pachymetry were performed in all cases. Age, medical treatment for ocular hypertension, sex, and retinal nerve fiber layer thickness were evaluated. The main indices of blood flow were also examined. Spearman correlation coefficients were used to explore the association between two continuous variables.

\section{Results}

A directly proportional significance regarding the correlation between central corneal thickness and radial peripapillary network was indicated in eyes with ocular hypertension $(\mathrm{p}=.036)$.

\section{Conclusions}

Central corneal thickness and radial peripapillary capillary density constitute two essential screening parameters for patients with ocular hypertension.

Review began 07/27/2021 Review ended 08/02/2021 Published 08/12/2021

\section{() Copyright 2021}

Kollia et al. This is an open access article distributed under the terms of the Creative Commons Attribution License CC-BY 4.0., which permits unrestricted use, distribution, and reproduction in any medium, provided the original author and source are credited.
Categories: Ophthalmology

Keywords: central corneal thickness, radial peripapillary capillary density, optical coherence tomography angiography, ocular hypertension, risk factors

\section{Introduction}

Ocular hypertension $(\mathrm{OH})$ is defined as a condition in which the intraocular pressure (IOP) is greater than 21 $\mathrm{mmHg}$ in one or both eyes, with no evident glaucomatous defect. Its prevalence in people older than 40 years is estimated to range from $3 \%$ to $10 \%$ [1-4]. High IOP is a risk factor for conversion to primary openangle glaucoma. Increased intraocular pressure, advanced age, myopia, thin cornea, and dark skin color are the main risk factor [5-11]. The mean central corneal thickness (CCT) is higher in eyes with ocular hypertension than in normal or glaucomatous eyes. As reviewed in the Ocular Hypertension Treatment Study (OHTS), CCT may affect the accuracy of applanation tonometry in the screening and clinical treatment of patients with glaucoma and ocular hypertension. Hence, it has been studied in terms of its direct interrelationship with ocular pressure [2,8-11]. The recently released technology of optical coherence tomography angiography (OCT-A) constitutes a novel, non-invasive imaging method that detects blood flow in the retina and optic nerve head. It uses variations in the intensity and/or phase properties of the OCT signals that result from the movement of blood over multiple B-scans to generate a high-resolution map of the microcirculation. Thus, OCT-A provides a quick and meticulous evaluation of retinal vascular structures. Specifically, the radial peripapillary capillary plexus was assessed as far as the optic nerve head [12-18]. The principal aim of our study was to substantiate any correlation between CCT and radial peripapillary capillary density (RPC) in patients with ocular hypertension in terms of early and thorough clinical assessment of this entity.

\section{Materials And Methods}

In this study, 135 eyes with ocular hypertension were examined. Data were collected prospectively during one year. All subjects were adults over 18 years of age. Patients (eyes) with the following conditions were 


\section{Cureus}

excluded from the study: anti-glaucoma treatment with more than one pharmaceutical agent, any type of treatment for ocular diseases, dense cataracts, conditions consistent with abnormal macular findings, signs of uveitis, previous complicated cataract surgery, previous vitreoretinal interventions, previous refractive operations, and selective laser trabeculoplasty procedures.

The same protocol was applied to all the cases. First, all patients were carefully examined clinically. They underwent medical history recording, visual acuity evaluation, slit-lamp examination, tonometry, and fundus examination. Afterwards, OCT-A of the optic disc (4.5 mm program) and ultrasound corneal pachymetry were performed in all eyes. AngioVue HD (Optovue, Fremont, CA) software was used for all angiography measurements.

With regards to the applied statistical analysis, normally distributed variables were expressed as means (standard deviation), while variables with skewed distribution were expressed as median (interquartile range). Qualitative variables were expressed as absolute and relative frequencies. Spearman correlation coefficients were used to explore the association between two continuous variables. All reported p-values were two-tailed. Statistical significance was set at $\mathrm{p}<0.05$, and analyses were conducted using SPSS statistical software version 22.0 (IBM Corp., Armonk, NY).

All methods were carried out in accordance with the relevant existing guidelines and medical regulations. All experimental protocols involved in the study included the actualization of clinical and screening examinations. Ethical approval was obtained from the General Assembly of Medicine School of National and Kapodistrian University of Athens, after evaluation by the Scientific Committee for Research Ethics of Athens Medicine School. Written informed consent was obtained from all participants.

\section{Results}

The sample consisted of 135 patients with a mean age of 62.1 years +/-13.1 SD (Table 1). Most patients (61.5\%) were women. In addition, $47.4 \%$ of the patients were under medical treatment with one antiglaucoma agent. The median C/D ratio was 0.40 (IQR: $0.30-0.50$ ). The means and median values of the Retinal Nerve Fiber Layer (RNFL), CCT, and RPC measurements are presented in Table 2. Total peripapillary, superior-hemi, and inferior-hemi values of RPC were significantly positively associated with total peripapillary, superior-hemi, and inferior- hemi values of RNFL, indicating that higher RPC density values were significantly associated with higher RNFL values (Table 3). Inside disc values of RPC density were not significantly associated with RNFL values. Higher total peripapillary, superior-hemi, and inferior-hemi RNFL values were significantly associated with higher CCT values $(\mathrm{p}=.004 ; \mathrm{p}=.009$, and $\mathrm{p}=.030$, respectively) (Table 4$)$. The C/D ratio was only significantly and positively associated with CCT $(\mathrm{p}=.036)$. Furthermore, higher total peripapillary values were significantly associated with higher CCT values $(\mathrm{p}=.050)$ (Figure 1$)$. Inferior RPC values tended to be positively associated with CCT values $(p=.055)$.

\begin{tabular}{|l|l|}
\hline Age (years), mean (SD) & N (\%) \\
\hline Sex & $62.1(13.1)$ \\
\hline Women & $83(61.5)$ \\
Men & $52(38.5)$ \\
Under medical treatment & $64(47.4)$ \\
C/D Ratio, median (IQR) & $0.40(0.30-0.50)$ \\
\hline
\end{tabular}

\section{TABLE 1: Patients' characteristics}

SD: Standard deviation; IQR: Interquartile range; C/D ratio: cup-to-disc ratio. 


\section{Cureus}

\begin{tabular}{|c|c|c|}
\hline & Mean (SD) & Median (IQR) \\
\hline Total RNFL & $106.2(9.9)$ & $106(99-111)$ \\
\hline Superior & $105.8(10.9)$ & $105(97-114)$ \\
\hline Inferior & $106.7(10.9)$ & $105(99-114)$ \\
\hline ССТ $\mu \mathrm{m}$ & 536.7 (31.1) & $534(521.5-552.9)$ \\
\hline Total PP & $51.1(3.6)$ & $51.2(48.7-53.9)$ \\
\hline Superior & $51.3(4.2)$ & $51.5(48.3-54.0)$ \\
\hline Inferior & $50.7(4.6)$ & $50.9(48.3-53.9)$ \\
\hline Inside disc & $50.5(5.9)$ & $51.1(46.8-54.5)$ \\
\hline
\end{tabular}

TABLE 2: Description of RNFL, CCT and RPC measurements

RNFL: Retinal nerve fiber layer; CCT: Central corneal thickness; RPC: Radial peripapillary capillary density; PP: Peripapillary; SD: Standard deviation; IQR: Interquartile range.

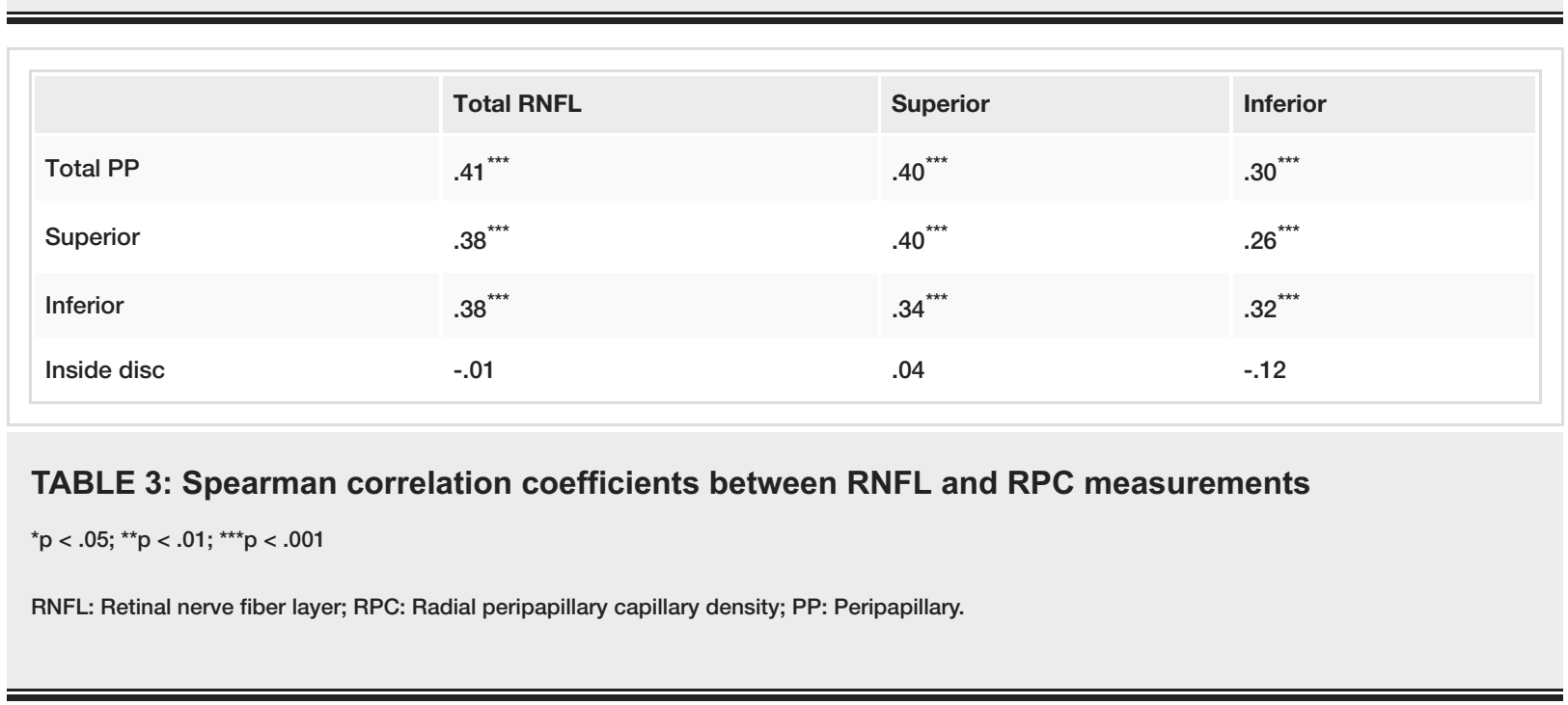




\section{Cureus}

\begin{tabular}{|c|c|c|}
\hline & ССТ - $\mu \mathrm{m}$ & C/D Ratio \\
\hline Total PP & $.17^{*}$ & -.02 \\
\hline Superior & .13 & .00 \\
\hline Inferior & .17 & -.05 \\
\hline Inside disc & .13 & -.06 \\
\hline Total RNFL & $24^{4 *}$ & .00 \\
\hline Superior & $22^{* *}$ & -.08 \\
\hline Inferior & $19^{*}$ & .06 \\
\hline C/D Ratio & $.18^{*}$ & \\
\hline
\end{tabular}

TABLE 4: Spearman correlation coefficients of RNFL and RPC measurements with CCT and C/D ratio

${ }^{*} \mathrm{p}<.05 ;{ }^{\star \star} \mathrm{p}<.01 ;{ }^{\star \star \star} \mathrm{p}<.001$

RNFL: Retinal nerve fiber layer; RPC: Radial peripapillary capillary density; CCT: Central corneal thickness; PP: Peripapillary; C/D ratio: cup-to-disc ratio.

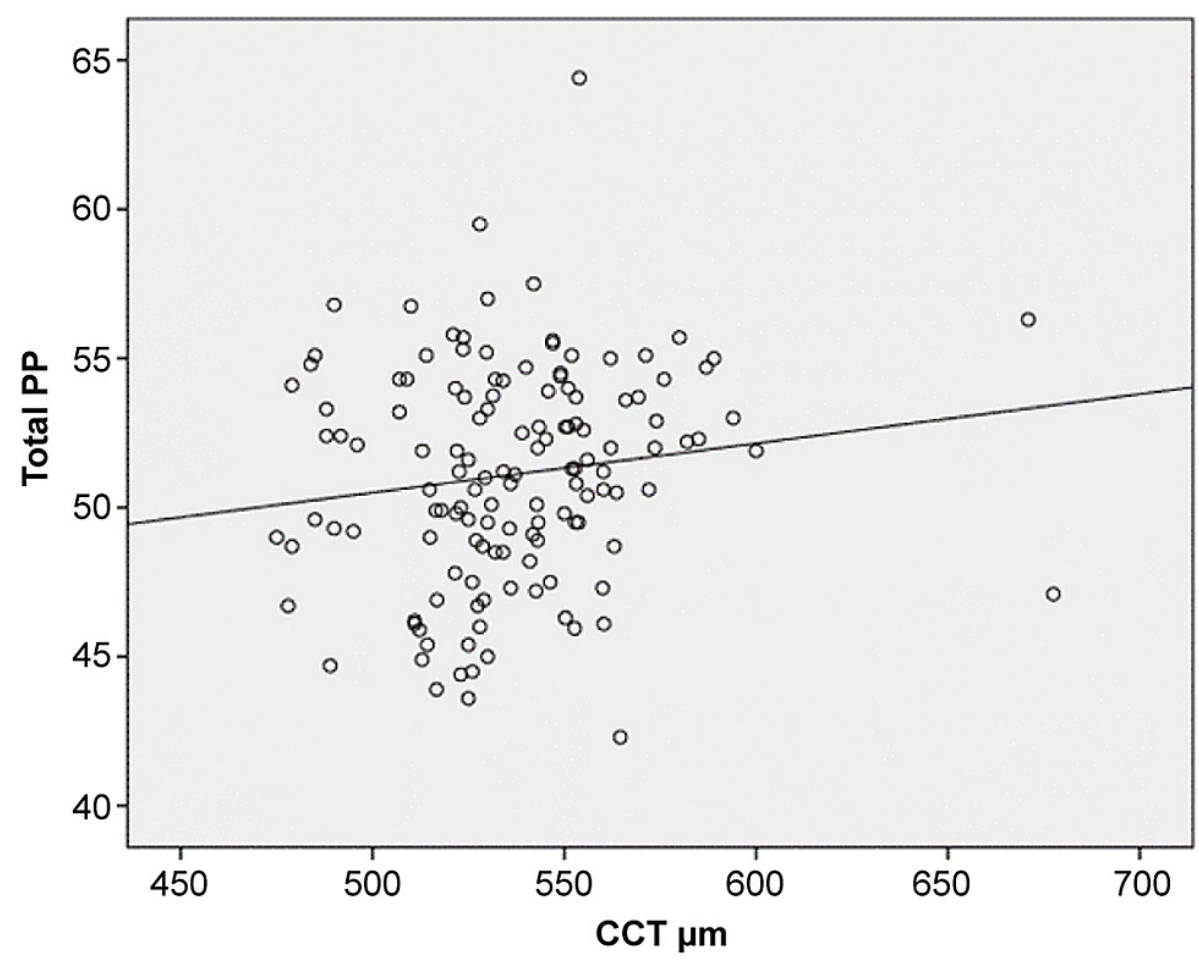

FIGURE 1: Correlation between total peripapillary capillary density (PP) and central corneal thickness (CCT)

Spearman correlation coefficient $(r)$ and $p$-value.

\section{Discussion}

$\mathrm{OH}$ has been an essential field of study. In the United States, its prevalence in non-Hispanic Whites over 40 years accounts for 4.5 percent, and increases up to 7.7 percent in 75 - to 79 -year-olds. In Latinos, the prevalence across ages is similar [18]. 
Risk factors and conditions related to $\mathrm{OH}$ have been meticulously studied. The Ocular Hypertension Treatment Study (OHTS), a multicenter randomized trial, remains the principal guiding light for all scientific approaches towards new immersive data. Its purpose was to evaluate the safety and efficacy of topical ocular hypotensive medical agents in delaying or preventing the onset of primary open-angle glaucoma (POAG) in individuals with elevated intraocular pressure (IOP) and no detectable glaucomatous damage.

The latest update of the OHTS indicates the baseline factors that predict the evolution of primary openangle glaucoma. These include older age, race (African American), sex (male), larger vertical cup-disc ratio, larger horizontal cup-disc ratio, increased intraocular pressure, higher Humphrey visual field pattern standard deviation, heart disease, and thinner central cornea (CCT) [1-9, 1, 18-20].

Additionally, the RNFL assessment is widely used to weigh the amount of structural loss in $\mathrm{OH}$ and glaucoma, since the retinal thickness measurements present significant thinner change in patients suspected for glaucomatous conditions [18-19].

However, ocular blood flow changes have been considered to play a key role in the onset and development of different retinovascular and optic nerve diseases. Thus, not only RNFL thickness but also the density of RPCs network may also be associated with optic nerve head parameters and possible glaucomatous damage [21].

Furthermore, the 'ISNT rule', referring to the normal optic disc rim width (inferior $\geqslant$ superior $\geqslant$ nasal $\geqslant$ temporal) has also been used as a tool in screening for and diagnosing such entities [22].

In our study, after the evaluation of all parameters, significant interconnection between two essential parameters for the diagnostic algorithm of ocular hypertension parameters was encountered. There is an important correlation between CCT and RPC density. Moreover, after individually approaching the collected data, the low percentage of RPC density in eyes with $\mathrm{OH}$ was statistically significant. Specifically, a lower proportion of RPC density in the inferior hemi-quadrant of the optic nerve head in these eyes could potentially be associated with parallel or future structural changes. As a result, the possibility of conversion to primary open-angle glaucoma would be enhanced as the "ISNT" rim pattern (neural rim width: inferior 》 superior $\geqslant$ nasal $\geqslant$ temporal) could be verified $[15,11,18-22]$.

\section{Conclusions}

In light of the above, the CCT and RPC density constitute two essential screening parameters for patients with ocular hypertension. After having taken all the aforementioned aspects into consideration, we can clearly conclude that the CCT and RPC density constitutes two associated parameters. We could assume that the newly introduced value of RPC density could be considered as an additional risk factor to those described in the OHTS. However, it would be interesting to examine this factor over a long-term period and with reference to scientific studies concerning primary open-angle glaucoma.

\section{Additional Information}

\section{Disclosures}

Human subjects: Consent was obtained or waived by all participants in this study. Scientific Committee for Research Ethics of Athens Medicine School issued approval Not applicable. All methods were carried out in accordance with the relevant existing guidelines and medical regulations. All experimental protocols involved in the study included the actualization of clinical and screening examinations. Animal subjects: All authors have confirmed that this study did not involve animal subjects or tissue. Conflicts of interest: In compliance with the ICMJE uniform disclosure form, all authors declare the following: Payment/services info: All authors have declared that no financial support was received from any organization for the submitted work. Financial relationships: All authors have declared that they have no financial relationships at present or within the previous three years with any organizations that might have an interest in the submitted work. Other relationships: All authors have declared that there are no other relationships or activities that could appear to have influenced the submitted work.

\section{References}

1. European Glaucoma Society: Terminology and Guidelines for Glaucoma. European Glaucoma Society, 2020.

2. Renard JP, Giraud JM, Crochelet O, Reda K, May I, Rigal-Sastourne JC, Maurin JF: Bilan en pratique. L'hypertonie oculaire isolée [Practical assessment. Ocular hypertension]. J Fr Ophtalmol. 2005, 28:13-16. 10.1016/S0181-5512(05)81159-3

3. Funk J: Ocular hypertension. What is it actually? (Article in German) . Ophthalmologe. 2011, 108:1005. 10.1007/s00347-011-2379-9

4. Lee BL, Wilson MR: Ocular Hypertension Treatment Study (OHTS) commentary . Curr Opin Ophthalmol. 2003, 14:74-77. 10.1097/00055735-200304000-00003

5. Gordon MO, Beiser JA, Brandt JD, et al.: The Ocular Hypertension Treatment Study: baseline factors that predict the onset of primary open-angle glaucoma. Arch Ophthalmol. 2002, 120:714-720.

10.1001/archopht.120.6.714

6. Hoffmann EM, Lamparter J: Differentiation of ocular hypertension (Article in German) . Ophthalmologe. 
2016, 113:715-728. 10.1007/s00347-016-0304-y

7. Sun YY, Chen WW, Wang NL: Diagnosis and treatment of ocular hypertension (Article in Chinese) . Zhonghua Yan Ke Za Zhi. 2016, 52:542-546. 10.3760/cma.j.issn.0412-4081.2016.07.016

8. Kass MA, Heuer DK, Higginbotham EJ, et al.: The Ocular Hypertension Treatment Study: a randomized trial determines that topical ocular hypotensive medication delays or prevents the onset of primary open-angle glaucoma. Arch Ophthalmol. 2002, 120:701-713. 10.1001/archopht.120.6.701

9. Boey PY, Mansberger SL: Ocular hypertension: an approach to assessment and management. Can J Ophthalmol. 2014, 49:489-496. 10.1016/j.jcjo.2014.06.013

10. Wang SY, Melles R, Lin SC: The impact of central corneal thickness on the risk for glaucoma in a large multiethnic population. J Glaucoma. 2014, 23:606-612. 10.1097/IJG.0000000000000088

11. Gordon MO, Kass MA: What we have learned from the ocular hypertension treatment study . Am J Ophthalmol. 2018, 189:xxiv-xxvii. 10.1016/j.ajo.2018.02.016

12. Ang M, Tan AC, Cheung CM, Keane PA, Dolz-Marco R, Sng CC, Schmetterer L: Optical coherence tomography angiography: a review of current and future clinical applications. Graefes Arch Clin Exp Ophthalmol. 2018, 256:237-245. 10.1007/s00417-017-3896-2

13. Kashani AH, Chen CL, Gahm JK, et al.: Optical coherence tomography angiography: a comprehensive review of current methods and clinical applications. Prog Retin Eye Res. 2017, 60:66-100. 10.1016/j.preteyeres.2017.07.002

14. Akil H, Falavarjani KG, Sadda SR, Sadun AA: Optical coherence tomography angiography of the optic disc; an overview. J Ophthalmic Vis Res. 2017, 12:98-105. 10.4103/2008-322X.200162

15. MacCormick IJ, Williams BM, Zheng Y, et al.: Accurate, fast, data efficient and interpretable glaucoma diagnosis with automated spatial analysis of the whole cup to disc profile. PLoS One. 2019, 14:e0209409. 10.1371/journal.pone.0209409

16. Lauermann JL, Eter N, Alten F: Optical coherence tomography angiography offers new insights into choriocapillaris perfusion. Ophthalmologica. 2018, 239:74-84. 10.1159/000485261

17. Alnawaiseh M, Lahme L, Eter N, Mardin C: Optical coherence tomography angiography: value for glaucoma diagnostics (Article in German). Ophthalmologe. 2019, 116:602-609. 10.1007/s00347-018-0815-9

18. American Academy of Ophthalmology: 2019-2020 BCSC: Basic and Clinical Science Course. American Academy of Ophthalmology, San Francisco; 2019.

19. Salmon JF: Kanski's Clinical Ophthalmology: A Systematic Approach. Elsevier, Edinburgh; 2020.

20. Argus WA: Ocular hypertension and central corneal thickness. Ophthalmology. 1995, 102:1810-1812. 10.1016/S0161-6420(95)30790-7

21. Ozcan Y, Ozcaliskan S, Balci S, Artunay O: The correlation of radial peripapillary capillary density measurements with optic nerve head morphology and retinal nerve fiber layer thickness in healthy eyes. Photodiagnosis Photodyn Ther. 2020, 32:102008. 10.1016/j.pdpdt.2020.102008

22. Moon J, Park KH, Kim DM, Kim SH: Factors affecting ISNT rule satisfaction in normal and glaucomatous eyes. Korean J Ophthalmol. 2018, 32:38-44. 10.3341/kjo.2017.0031 